\title{
Facile synthesis of nickel nanowires with controllable morphology
}

\author{
Ying Ying Kong, Suh Cem Pang*, Suk Fun Chin \\ Department of Chemistry, Faculty of Resource Science and Technology, Universiti Malaysia Sarawak, 94300 Kota Samarahan, Sarawak, Malaysia
}

\section{A R T I C L E I N F O}

\section{Article history:}

Received 17 September 2014

Accepted 25 November 2014

Available online 4 December 2014

\section{Keywords:}

Particles

Nanosize

Magnetic materials

Metals and alloys

Microstructure

\begin{abstract}
A B S T R A C T
Nickel nanowires of controllable morphology were synthesized by a facile wet chemical reduction method using hydrazine hydrate as the reducing agent. Synthesis parameters such as reaction temperature, concentration of nickel ions, and $\mathrm{pH}$ of reaction medium were observed to have significant effects on the morphology of nickel nanowires formed. Only randomly aggregated nanoparticles were obtained at the reaction temperature of $30^{\circ} \mathrm{C}$. However, visibly defined nickel nanowires of longitudinally aligned nanoparticles, and fully transformed, well-defined long and slender nanowires were obtained at reaction temperatures of $60^{\circ} \mathrm{C}$ and $120^{\circ} \mathrm{C}$, respectively. Higher reaction temperature led to the formation of nickel nanowires of decreasing mean diameters, whereas increasing initial concentration of nickel ions resulted in the formation of nickel nanowires of larger mean diameters. Besides, welldefined nickel nanowires were formed at the reaction medium $\mathrm{pH}$ of 4-5. Henceforth, the morphology of nickel nanowires could be modulated readily via precise control of the synthesis parameters such as reaction temperature and initial concentration of nickel ions. Nickel nanowires of tailored morphology could potentially be useful for the fabrication of nanowire-based electronic or electrochemical devices. (c) 2014 Published by Elsevier B.V.
\end{abstract}

\section{Introduction}

Nanowires, nanotubes and nanorods of magnetic materials such as iron, cobalt, and nickel are attracting increasing attention from researchers worldwide. These 2-D nanostructures have been recognized as important building blocks for the fabrication of electronic devices, magnetic sensors, catalysts, electrode materials and so forth $[1,2]$. Several common methods have been used by researchers to synthesize nickel nanowires which include hydrothermal, laser ablation, chemical vapor deposition (CVD), and template synthesis [3]. Since physical and chemical properties of nanostructures were significantly affected by the morphology of nanowires, extensive researches are being conducted to afford morphological control of nanowires during synthesis [4].

Template synthesis is the most commonly used method for synthesizing nanowires due to its versatility in controlling of the growth and dimension of nanowires. The template synthesis method has been used to prepare nickel nanowires of regular and controllable rod sizes. However, template synthesis is not cost-effective and unsuitable for large scale production [5]. Microemulsion is another synthesis method being used for synthesizing nanowires of controllable morphology. Nickel nanorods with a diameter of $8-10 \mathrm{~nm}$ and length of 100-200 $\mathrm{nm}$ had been successfully prepared by the

\footnotetext{
* Corresponding author.

E-mail address: scpang@frst.unimas.my (S.C. Pang).
}

reduction of nickel chloride $\left(\mathrm{NiCl}_{2}\right)$ with hydrazine hydrate in water/ butanol/potassium oleate/kerosene microemulsion [6]. Synthesis of nanowires in the aqueous phase is the preferred approach as it is non-flammable, cheap, environmental friendly, safe and feasible for large scale production [7]. Nickel nanowires were also prepared by the template-free method which entailed chemical reduction in the presence of a magnetic field for directing the structure of nanowires [8]. In this study, nickel nanowires were prepared by a facile wet chemical reduction method using hydrazine hydrate as the reducing agent. The effects of reaction temperature, initial concentration of nickel ions, and $\mathrm{pH}$ of reaction medium on the morphology of nickel nanowires formed were investigated.

\section{Experimental}

Materials: All chemicals were of analytical grade and used without further purification. Nickel chloride-6-hydrate $\left(\mathrm{NiCl}_{2} \cdot 6 \mathrm{H}_{2} \mathrm{O}\right)$ and ethanol were purchased from Hamburg Chemical $\mathrm{GmbH}$, ethylene glycol $\left(\mathrm{HOCH}_{2} \mathrm{CH}_{2} \mathrm{OH}\right)$, isopropanol were purchased from MERCK, and hydrazine hydrate $98 \%\left(\mathrm{H}_{2} \mathrm{NNH}_{2} \cdot \mathrm{H}_{2} \mathrm{O}\right)$ was purchased from ALDRICH.

Sample preparation: Nickel nanowires were synthesized by the wet chemical reduction method [9] with some modifications. In this synthesis, $\mathrm{NiCl}_{2} \cdot 6 \mathrm{H}_{2} \mathrm{O}$ in ethylene glycol as a solvent was reduced by hydrazine hydrate to form nickel nanowires. Typically, predetermined volume of $1.0 \mathrm{M} \mathrm{NiCl}_{2} \cdot 6 \mathrm{H}_{2} \mathrm{O}$ aqueous solution was 